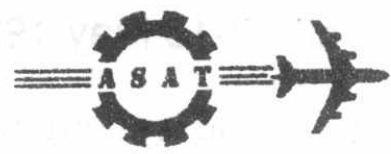

\title{
MECHANICAL ALIGNMENT USING LASER DIODES AND SINGLE ELEMENT PHOTO-DETECTORS; RESOLUTION ENHANCEMENT
}

\author{
M.M. AHMED*, A.S. GERGES**, E.A. MEHANNA***
}

\begin{abstract}
This paper studies the use of laser diodes and single element photo-detectors in mechanical alignment. Considering Gaussian beam theoretical model, the effect of laser beam diameter is studied using computer simulation. Optimum conditions that give the highest sensitivity are proved to be where:

(i) the laser beam diameter matches the diameter of the sensitive area of the detector.

(ii) the laser beam waist is located at the sensitive area of the detector.
\end{abstract}

The photo-detector with two-complement sensitive area has been studied as a better replacement of a single area photodetector. It was proved that the use of this type of detectors, with all of the other parameters unchanged, doubles the alignment sensitivity.

Finally, an experimental verification has been performed which gives a good agreement with the results obtained by computer simulation, where an alignment sensitivity of $\pm 0.3558 \mu \mathrm{m}$ was obtained.

\section{INTKRODUCTION}

The development of laser is considered to be one of the major innovations in enormous number of applications. One of the most important applications of lasers is alignment, where the laser beam is used as a reference axis to align a given mechanical system[1]. A visible, $\mathrm{He}-\mathrm{Ne}$, or near infrared, diode laser, output beam is used together with suitable photodetector to align the constituents of the system relative to the beam.

* M. Sc., Dep. Of Physics, M.T.C. Cairo, Egypt. ** Associate professor, Dep. Of Physics, M.T.C. Cairo, Egypt. *** Associate professor, Dep. Of Physics, M.T.C. Cairo, Egypt. 
Optical detector is used to align the whole system relative to the laser beam. The accuracy which can be achieved in optical alignment is often limited by laser beam shape, diameter, type, size of the photodetector, and noise level at the detector's output. Theoretically, a laser beam is often thought to be equivalent to a straight weightless thread of unlimited extent but in practice it has a finite size and divergence that can be controlled by some special optics. To a good approximation, the laser output beam (in its lowest order mode $\mathrm{TEM}_{\mathrm{OO}}$ ) is approximated as a Gaussian beam , where the irradiance $I(r)$ of the beam, in terms of its axial value $I_{c}$, is given by [2]:

$$
I(r)=\frac{I_{c}}{\sqrt{2 \pi}} e^{-\left(2 r^{2} / w^{2}\right)}
$$

Where $r$ is the radial distance from the axis and $w$ is the spot size of the beam. At $r=w$, the irradiance is reduced to $1 / e^{2}=0.135$ of the central value. As the beam propagates in a direction $z$, the spot size varies according to the expression [2]:

$$
w(z)=w_{0} \sqrt{\left[1+\left(z / z_{0}\right)^{2}\right]}
$$

in which $z_{0}=\pi\left(w_{0}\right)^{2} \lambda$, where $w_{0}$ is the minimum spot size, known as the beam waist and $\lambda$ is the wavelength. The radius of curvature $\rho$ of the wavefront is given by [3]:

$$
\rho(z)=z+\left(z_{0}^{2} / z\right)
$$

As expected, both the diameter of the laser beam and its divergence angle ( known from the value of $\rho$ ) change from one position to another along the $z$-axis [4]. One target of this study is to determine the optimum beam size, and the locations of both the laser beam and the detector with respect to the system in order to achieve the highest possible accuracy.

There are many sources of noise, in the photodetector output, such as shot noise, background radiation noise, Johnson noise, dark current noise, and 1/f noise [5,6]. It causes random fluctuations in the output that cannot be distinguished from the signal and hence limits the system's accuracy. The photodiodes are either single element or multi-element, in which more than one sensitive area are integrated in the same photodiode. This paper studies the use of the single element and two complement elements photodiodes while the multi-element photodiodes will be discussed in a future work. 


\section{OPTIMIZATION USING COMPUTER SIMULATION}

Consider a laser beam propagating along $\mathrm{z}$-axis where a photodiode is placed at a distance $(z)$ in front of the laser beam, with its center coinciding with the $z$-axis. To simulate a miss-alignment ( $r$ ), between the centers of the photodiode and the Gaussian beam , consider the following: (i) The laser is displaced a distance ( $x$ ) perpendicular to the $\mathrm{z}$-axis ( position miss-alignment), in this case $\mathrm{r}=\mathrm{x}$, (ii) The laser is rotated a small angle $(\theta)$ around the origin (angular miss-alignment), hence $r=\theta z$. For a single element photodiode, the laser beam diameter (w) is either greater than, equal to, or smaller than the diameter of the photodiode sensitive area (D).

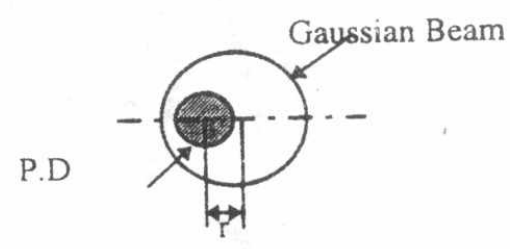

(a)

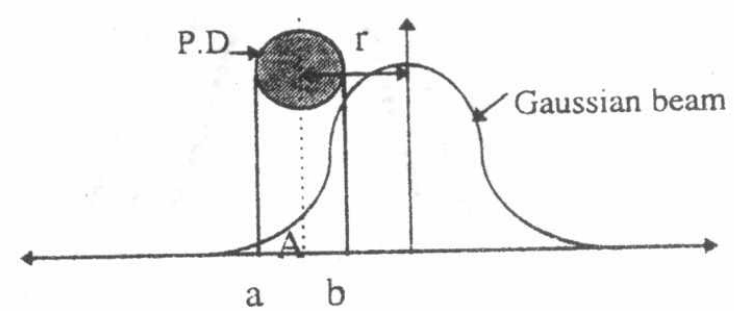

(b)

Fig.1. Optical power received by the photodiode.

In Fig.1a,b, the dashed area A represents the part of optical power, of the laser Gaussian beam, that falls on the photodiode. Considering a photodiode homogeneity the output voltage (current) of the photodiode is given by : $V_{d}=R A$, where $R$ is the responsivity of the photodiode. Substituting for A, then:

$$
\mathrm{V}_{\mathrm{d}}=\mathrm{RI}_{\mathrm{i}} \int_{\mathrm{a}}^{b} \frac{\mathrm{e}^{-\left(2 \mathrm{r}^{2} / \mathrm{w}^{2}\right)}}{\sqrt{2 \pi}} \mathrm{dr}
$$

Trapezoidal method, for numerical integration, has been used to calculate numerical values of the above integration. This simulates the expected variations of the phtodiode output as a function of miss-alignment ( $r$ ). These results are shown in Fig.2, for the cases of $D<w, D=w$ and $D>w$ respectively.

Fig.2, shows that for $\mathrm{D}<\mathrm{w}$, the maximum output is obtained when the missalignment ( $r$ ) is zero (perfect alignment), and as ( $r$ ) increases, or decreases the output voltage decreases. For all the factors unchanged, if $\mathrm{D}=\mathrm{w}$ (Fig.2.), a similar curve for the output is obtained but with much higher slope, specially near $r=0$. Moreover at $r=0$, the output voltage of the detector is nearly twice that obtained in the first case 
which indicates that more optical power is received by the photodiode. In the third case, when $D>w$, although the output is high as that obtained in the second case, it shows a flat region at the position of perfect alignment which describes the case where the whole beam falls inside the sensitive area of the photodiode. Within this region, the system is blind and can not tell where is the position of the laser beam with respect-to the photodiode.

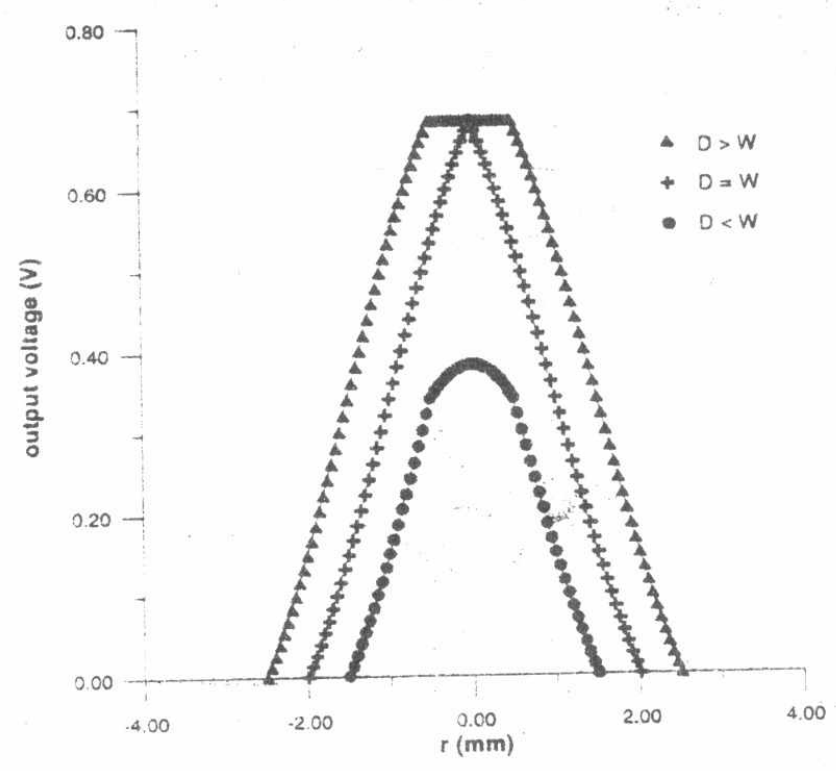

Fig.2. The output voltages of a single element photo diode versus the miss-alignment (r).

\section{ALIGNMENT SENSITIVITY \& SYSTEM RESOLUTION}

The sensitivity $\mathrm{S}(\mathrm{r})$ of determining the miss-alignment is defined as the rate of change. of the output voltage with respect to (r). GRAMS/386 software [7] for numerical differentiation was used to calculate the sensitivities for the three cases in the vicinity of the position of perfect alignment $(r=0)$. The results show that the maximum sensitivity is obtained in the case of beam- photodiode matching, at $D=w$.

To study the effect of noise level in the determination of the minimum detectable missalignment (system resolution $\Delta r$ ), consider that the output voltage is superimposed with a noise of level $=( \pm N)$. Then the corresponding position of perfect alignment is $(r=0 \pm \Delta r)$. It can be proved that $\Delta r=N / S$, which illustrate the importance of: (i) minimizing the noise level at the photodetector output, this shows the importance of laser beam modulation to avoid the high level of noise floor at low frequency. (ii) Optimizing the system to obtain at the highest possible sensitivity . 


\section{ALIGNMENT USING A TWO-COMPLEMENT PHOTODIODE ARRAY}

This array consists of a circular photodiode with diameter $\mathrm{D}$ enclosed by a second photodiode of large sensitive area, integrated in one case. The two complement outputs of the photodiodes are commonly taken through a differential amplifier of output $V_{0}=G\left(V_{1}-V_{2}\right)$, where $G$ is the gain of the amplifier, $V_{1}$ and $V_{2}$ are the output voltages of photodiode 1 and 2 respectively, which are proportional to the areas $A_{1}$ and $\mathrm{A}_{2}$ ( in Fig. 3 ).
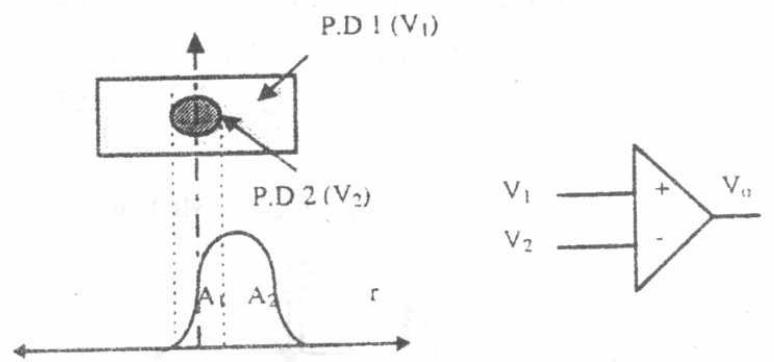

Fig.3. The use of two-complement photodiode array in alignment

Using an algorithm, similar to that used with the single element photodiode, and for $\mathrm{G}=1$, the outputs are obtained, as shown in Fig. 4 . The improvements obtained by

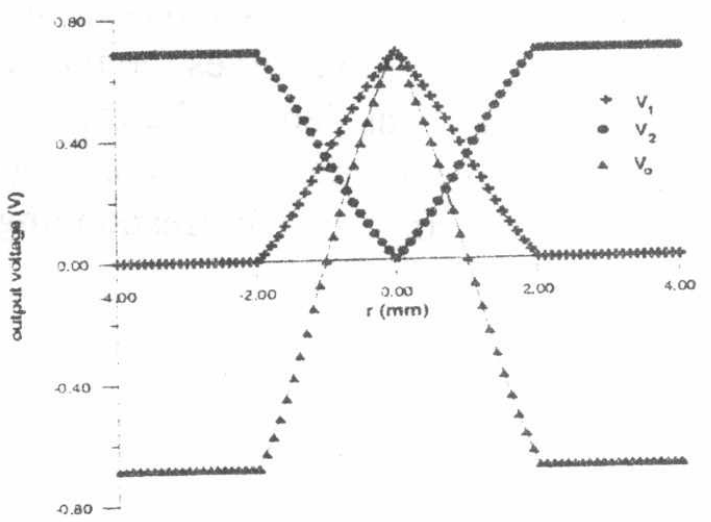

Fig.4. The output voltages of the elements of the two-complement photodiode array

$\left(\mathrm{V}_{1}, \mathrm{~V}_{2}\right)$ and the total output voltage $\left(\mathrm{V}_{\mathrm{o}}\right)$.

using such a device results from the following : (i) Very small noise level in the output signal, since most of the noise associated with the two outputs $\mathrm{V}_{1}$ and $\mathrm{V}_{2}$ are common. This common mode noise is rejected at the output of the differential amplifier, (ii) The sensitivity of the device is nearly two times better than that obtained by a single photodiode working in the same environment. Hence the system resolution is enhanced and a much better system accuracy is expected. 


\section{EXPERIMENTAL VERIFICATION}

The set up shown in Fig.5, was used for experimental verification. A laser diode of wavelength $\lambda=670 \mathrm{~nm}$, and output power of $1 \mathrm{~mW}$, was sinusoidally modulated at 2 $\mathrm{kHz}$ to avoid the high level of the low frequency noise. The laser diode was fixed on an optical bench in front of the beam shaping optics. At a distance of about $60 \mathrm{~cm}$ from the laser, a PIN photodiode (with $1 \mathrm{~mm}^{2}$ sensitive area), was mechanically fixed on an $\mathrm{x}-\mathrm{y}-\mathrm{z}$ micro translation stage (of step accuracy $\pm 5 \mu \mathrm{m}$ ). The beam shaping optics were adjusted to nearly match the diameters of the beam and the sensitive area of the photodiode. The output of the photodiode is taken directly to the measuring

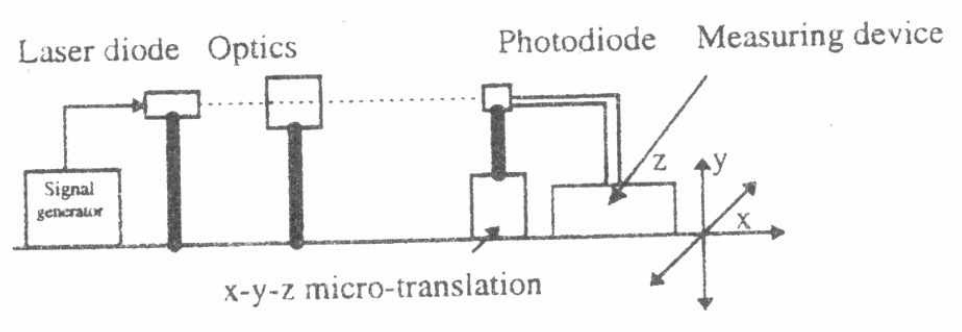

Fig.5. The block diagram of the experimental set up.

devices. At first, the micro-translation stage is adjusted at the position of perfect alignment $(r=0)$, at which the photodiode gives the maximum output voltage. The corresponding values of $\mathrm{x}$ and $\mathrm{y}$ are considered to be the system origin $(\mathrm{x}=0, \mathrm{y}=0)$. Next, different values of miss-alignment were produced, by moving the controls of the micro-translation stage, and measuring the corresponding values of the output voltage, as shown in Fig.6.

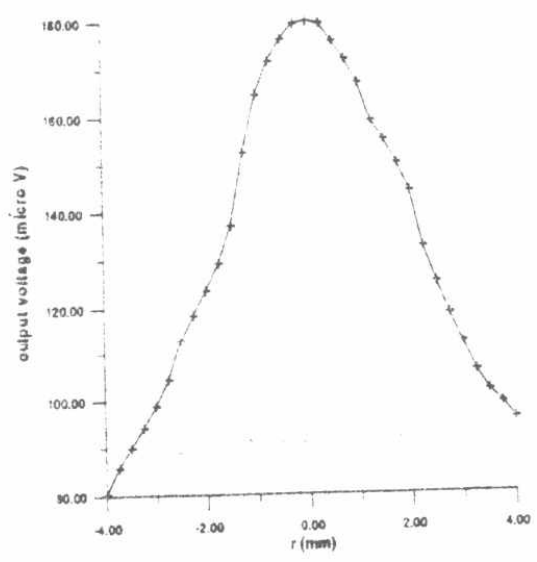

Fig.6. Experimental output voltage for a single photodiode.

Fig.7 indicates the level of the output signal, at perfect alignment, compared with the noise floor of the system as measured by the spectrum analyzer. The noise floor of the 
system output at $2 \mathrm{kHz}$ is very low $\approx 4 \mu \mathrm{V}$, which indicates the advantage of modulating the laser diode. From the experimental data, we can estimate a numerical value of the system resolution $\Delta r= \pm 0.3558 \mu \mathrm{m}$.

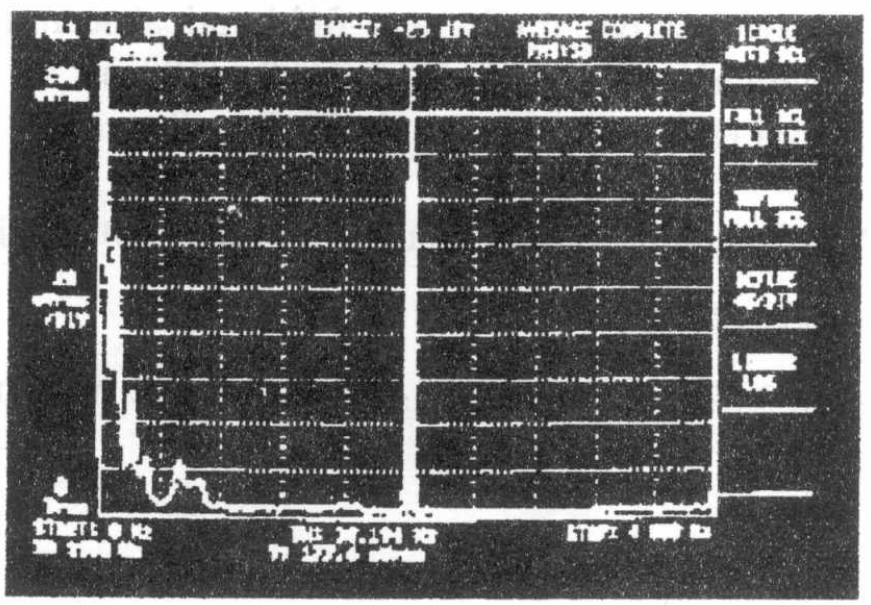

Fig.7. The photodiode output voltage as monitored by a spectrum analyzer at perfect alignment $(r=0)$ compared with the noise floor.

\section{CONCLUSIONS}

The low-power lasers are commonly used for both position and angular alignment for mechanical systems. The laser beam profile, noise level in the system, and the type of photodetector affect the accuracy of alignment. For single element photodiode and a two-complement photodiode array, we have proved that the optimum conditions are obtained when the laser beam and the photodiode sensitive area are matched together. Moreover, it has been proved that better performance is obtained when the beam waist is located at the detector, since the noise level on the detector output is reduced. The use of the two-complement photodiode array, as a better replacement for the single element photodiode, is studied. It doubles the sensitivity and reduces the noise level which enhances the system resolution. The theoretical study is confirmed by the experimental results which were of good match with that expected by the computer simulation.

\section{REFERENCES}

[1] Kingslake R., "Optical System Design", Academic Press Inc., New York, (1983). [2] Williams D. C., "Optical Methods In Engineering Metrology", Cambridge University Press, London, (1993).

[3] Boaz Salik, et al., " One dimensional beam shaping", J. of the optical society of America A, Vol. 12, No. 8, pp 1702-1706, (1995). 
[4]Joshua L. Kann, Tom D. Milster, Fred F. Froehlich, Richard W. Ziolkowski, and Justin B. Judkins, " Linear behaviour of a near-field optical scanning systems", J. of the optical society of America A, No. 8, pp 1677-1682, (1995).

[5] McKinley W. G., Yura H. T., and Hanson S. G., Opt. Soc. Am., No. 5, pp 333-335 (1988).

[6] Chin-Lin Chin, "Elements of optoelectronics and fiber optics", R. R. Donnelley \& Sons Company, New York, (1996).

[7] GRAMS/386, version 3.01C, level 1, 1991-1994, Galactic Industries Corporation. 